\title{
Reducción del tamaño de la clase de Inglés empleando Blended Learning como estrategia de aprendizaje
}

\section{Clayton Carrasco Grijalva}

Profesor de Inglés e investigador de la Universidad de Guayaquil, clayton.carrascog@ug.edu.ec, clay_u2@hotmail.com

\begin{abstract}
The present work aims to detail the occurrences and results obtained from a research project achieved with first semester students in the subject of English I that is part of the curriculum of the career of Chemistry and Pharmacy in an Ecuadorian University. The main objective targeted the class size reduction, which stands for the reduction of student-teacher ratio that also implied the reduction of the class time length without affecting the learning quality. To achieve this objective the course of study was adapted to the Blended Learning mode and supported by the application NOMON PILLBOOK and NOMON CISE as an educational technology tools that were developed by the researcher.
\end{abstract}

Keywords: class size, blended learning, educational technology tool, learning quality, innovation, ubiquitous learning

\begin{abstract}
Resumen
El presente trabajo detalla incidencias y resultados obtenidos de un proyecto de investigación realizado con estudiantes del primer semestre en la asignatura de inglés I que forma parte de la malla curricular de la carrera de Química y Farmacia en una universidad ecuatoriana. El objetivo central de la intervención fue reducir el tamaño de la clase; es decir reducir el ratio de alumnos por profesor, lo que implicó reducir también la duración presencial de dichas clases sin afectar la calidad del aprendizaje. Para lograr este objetivo se empleó la modalidad Blended Learning y como herramientas tecnológicas educativas de apoyo la aplicaciones NOMON PILLBOOK y NOMON CISE de desarrollo propio.
\end{abstract}

Palabras clave: tamaño de la clase, aprendizaje fusionado, herramienta tecnológica educativa, calidad del aprendizaje, innovación, aprendizaje ubicuo

\section{Introducción}

Para distintos expertos la reducción del tamaño de la clase es un factor de incidencia clave que sumado a otros factores metodológicos permite la mejora en la adquisición del aprendizaje generando un ambiente de aprendizaje más activo. Hughes (2005) manifiesta que la reducción del tamaño de clase permite a los profesores emplear mejor pedagogía así 
como la adopción de cambios de contexto que incluyen diversidad, inclusión y evaluación. Como plantea el autor, la conducta del estudiante mejora en clases pequeñas y el aprendizaje se convierte en una tarea de mayor responsabilidad y compromiso para éste. De acuerdo a diversos autores, la efectividad de este proceso responde a diferentes situaciones. Mayer \& Peterson (1999) destacan como una de estas situaciones al tipo de organización que tenga la institución educativa. Sin embargo, Calero \& Escardibul (2007) mencionan que cuando se comparan diferentes estudios en torno a la reducción del tamaño de clase, las conclusiones no son coincidentes. Biddle \& Berliner (2008) corroboran este criterio señalando que existen grupos en favor y otros en contra de que clases más pequeñas conduzcan a los estudiantes a mayor rendimiento estudiantil.

En cuanto a la reducción del tamaño de la clase, hay dos aspectos importantes que deben considerarse según Chingos (2013): uno es el ratio o relación de alumnos por profesor y otro el tamaño promedio de las clases. El autor menciona que proyectos como el Tennessee STAR (Mosteller, 1995) estiman una reducción ideal de entre 20 a 15 alumnos por profesor, de clases que fluctuaban en inicio entre 35 a 30 alumnos por profesor.

El informe "Education at a Glance" (OECD, 2011) cita como aspectos de incidencia que las clases pequeñas o reducidas permiten a profesores enfocarse más en las necesidades particulares de cada estudiante, situación que permite suponer que éstas tienen mayor costo que las tradicionales, por ende requieren de profesorado mejor entrenado, mejor calidad de material didáctico, mayores recursos tecnológicos para el aula, etc.

Por otro lado, hablar de aprendizaje ubicuo no solo se refiere al acceso que se tiene a la información desde cualquier lugar, sino como lo describe Burbules (2012), se trata de tener el control de dónde, cuándo, cómo y por qué cada individuo está aprendiendo. El Aprendizaje Fusionado conocido también como Blended Learning cumple con esta lógica de darle dicho "control" al estudiante. Vera (2008) lo define como un modelo híbrido por medio del cual tutores hacen uso de sus metodologías presenciales en el aula y paralelamente potencian el desarrollo de sus temáticas empleando una plataforma virtual, la que a la vez está dotada de un sinnúmero de recursos entre los que pueden encontrarse los juegos serios y otros asociados a la gamificación. Singh (2003) sugiere que las organizaciones deben emplear Aprendizaje Fusionado en función de proveer el contenido correcto a la gente correcta en el tiempo correcto.

En lo que respecta al aprendizaje de inglés en las universidades, Hans \& Hans (2015) indican que los programas por lo general son del tipo EFL (inglés como lengua extranjera) o ESP (inglés para fines específicos). Muchos de los currículos para la enseñanza de inglés en las Universidades latinoamericanas incluidas las ecuatorianas, de acuerdo a Cronquist y Fiszbein (2017) emplean el Marco Común Europeo de Referencia para las Lenguas (Instituto Cervantes, 2012) como estándar del aprendizaje. Al ser el aprendizaje de inglés de corte técnico con fines de profesionalización más que de corte social, como lo señalan Garrido y Pernas (2005) surgen conflictos cuando los estudiantes carecen de los conocimientos base necesarios para seguir un programa de este tipo. Mora (2015) agrega como factor negativo y recurrente la falta del empleo de las TIC.

(cc) EY-NC-ND 2018, Universitat Politècnica de València 


\section{Objetivo}

El objetivo de esta innovación fue reducir el tamaño de una clase presencial en la asignatura de Inglés I, considerando como factores para dicha reducción: el ratio de alumnos por profesor y la duración del tiempo presencial de la clase; y que esta combinación de factores no afecte la calidad del aprendizaje. El estudio planteó tres hipótesis para su validación.

En cuanto al método de comprobación, el estudio se enfocó en comparar el desempeño académico en la asignatura de Inglés I durante el primer ciclo semestral del año lectivo 2017-2018 de un grupo de estudiantes en modalidad presencial (grupo de control sin intervención) y un grupo de estudiantes en modalidad Blended Learning (grupo experimental con intervención) de 40 estudiantes cada uno.

Se plantearon las hipótesis:

a) Puede reducirse el tamaño de la clase de Inglés empleando la modalidad Blended Learning sin afectar la calidad del aprendizaje.

b) La modalidad Blended Learning puede reforzar de mejor forma el aprendizaje del idioma Inglés en comparación al aprendizaje presencial.

c) El uso de una herramienta tecnológica de apoyo permite reducir el tiempo presencial de una clase de Inglés sin afectar la calidad del aprendizaje.

\section{Desarrollo}

El enfoque metodológico empleado fue de tipo cuantitativo, dado a que este tipo de metodología permite entender a modo de tendencia comparativa las connotaciones más relevantes, así como los resultados y cambios se logran a partir de la innovación.

Los participantes del estudio correspondieron a estudiantes del primer semestre cuyas edades fluctuaron entre los 18 y 22 años. El tamaño de la muestra fue de 80 estudiantes. Se tuvo de contexto a la Facultad de Ciencias Químicas de la Universidad Estatal de Guayaquil.

Como fundamento pedagógico de la innovación fue adoptado el modelo Flipped Classroom o clase inversa que permitió establecer el criterio de emplear el tiempo presencial para la práctica y evaluación de contenidos dejando las actividades tipo lección tutorial para el tiempo virtual del que los estudiantes pudieran disponer en función de sus capacidades y necesidades.

Para la reducción del tamaño de la clase se adoptó la siguiente estrategia: el grupo experimental antes de la innovación solía ser 1 grupo de 40 estudiantes con una frecuencia de 4 horas por semana en modalidad presencial empleando material didáctico tradicional. Aplicando la innovación de la clase de tamaño reducido, esto cambió para tener 4 grupos en lugar de solo 1 empleando la modalidad Blended Learning, con una frecuencia de 1 hora de clase presencial por semana para cada grupo pero haciendo uso de una herramienta tecnológica de apoyo para el aprendizaje autónomo y considerando un mínimo de 3 horas 
Reducción del tamaño de la clase de inglés empleando Blended Learning como estrategia de aprendizaje

por semana. El grupo de control se mantuvo en el formato de clase presencial, lo que quiere decir 1 grupo de 40 estudiantes con una frecuencia de 4 horas de clase presencial por semana.

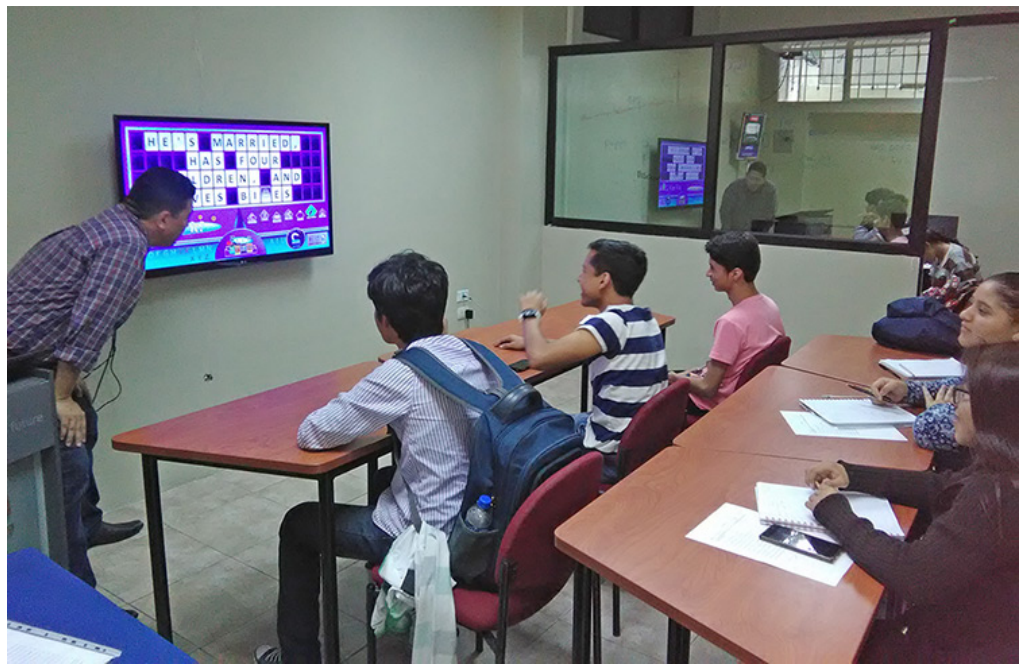

Fig. 1 Clase reducida, grupo experimental

Tanto el grupo experimental como el grupo de control recibieron los mismos contenidos en sus sesiones de clase de acuerdo al silabo de la asignatura. Cada grupo tuvo un profesor diferente, ambos profesores de la misma universidad y calificados para la enseñanza del idioma inglés.

En el caso del grupo de control que empleó modalidad de clase presencial, hizo uso de material didáctico tradicional como libros de texto, material de audio, etc., en concordancia con los contenidos y objetivos curriculares del silabo de la asignatura. El profesor de este grupo empleó un salón de clase con capacidad para 40 estudiantes acondicionado con un pizarrón y sistema de proyección para diapositivas.

En el caso del grupo experimental que empleó modalidad de clase Blended Learning, no hizo uso de material didáctico tradicional como lo hizo el grupo de control, en su lugar empleó la herramienta tecnológica de apoyo denominada NOMON PILLBOOK. Esta herramienta es una aplicación web que hace las veces de un libro digital que incluye en lecciones semanales las siguientes secciones: a) vocabulario incluida la práctica con ejercicio de contextualización, b) lección tutorial en video que incluye práctica con ejercicios de uso de lenguaje, c) dialogo para práctica oral y de comprensión auditiva, d) videos sobre el uso de verbos regulares y regulares; y e) panel de ejercicios orales para la pronunciación. Todos los contenidos de este recurso fueron personalizados en concordancia con los contenidos y objetivos curriculares del silabo de la asignatura. Les fue informado a los estudiantes de este grupo que debían realizar las actividades y seguir los contenidos fuera del tiempo de clase donde ellos mejor lo estimen (en sus casas o en la misma universidad, pero a través de sus computadoras personales) y en el tiempo que ellos establezcan, reflexionando en que el fundamento base del método era cumplir con los 
objetivos de aprendizaje de cada lección que en todos los casos se tradujo en el desarrollo de una determinada competencia o habilidad.

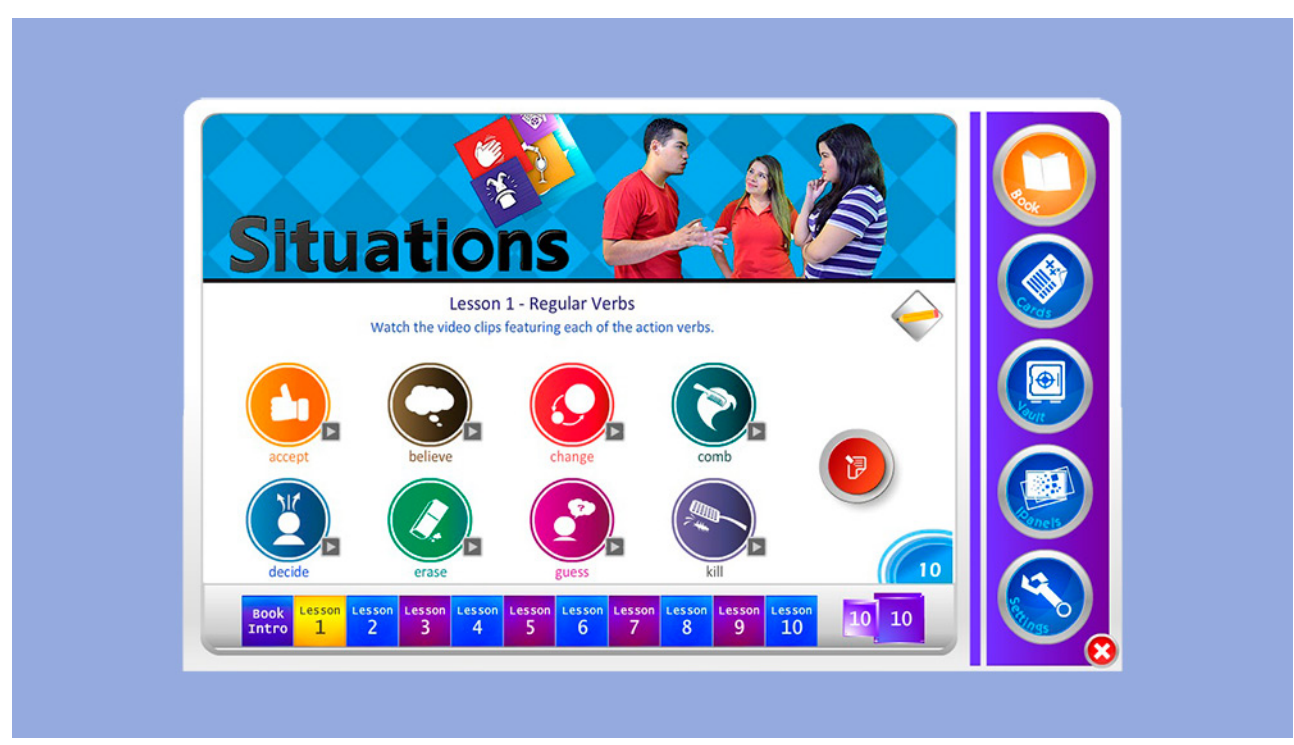

Fig. 2 Aplicación NOMON PILLBOOK

Para la parte presencial de una hora de clase de los 4 grupos de 10 estudiantes de las clases reducidas en la modalidad Blended Learning se destinó este tiempo a la evaluación y practica de los contenidos que los estudiantes previamente habían estudiado por medio de la aplicación NOMON PILLBOOK. El profesor para este acometido contó con la aplicación NOMON CISE que es complemento de la aplicación NOMON PILLBOOK. NOMON CISE muestra los contenidos de NOMON PILLBOOK pero en formato de juegos serios y actividades de ejercitación, además incluye diferentes funciones como asignar turnos, mostrar gráficos, evaluar ejercicios, etc. NOMON CISE a través de una pantalla de proyección de 48' muestra sus contenidos como si fuese un pizarrón inteligente en el que pueden hacerse anotaciones. El profesor de este grupo contó con un salón de clase especial adaptado para 10 estudiantes y acondicionado de acuerdo a los requerimientos de esta metodología. 


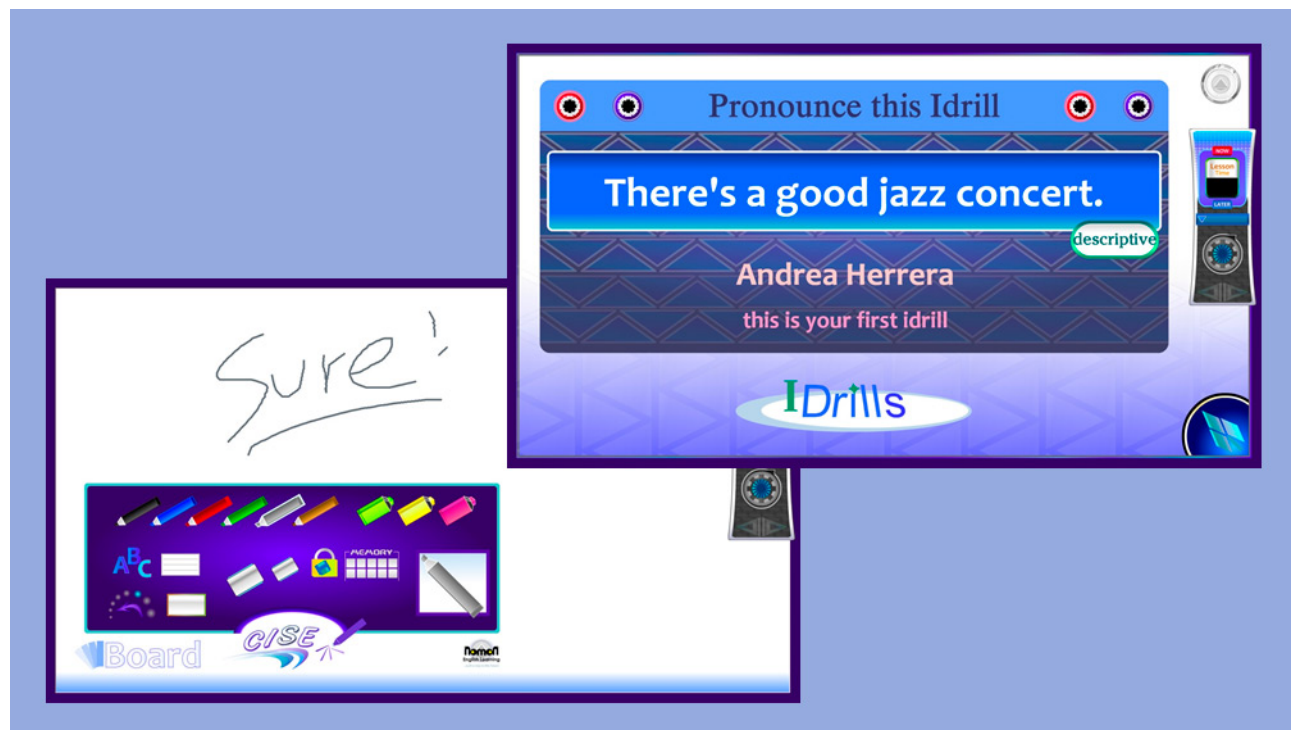

Fig. 3 Aplicación NOMON CISE

El rediseño de la unidad que propuso esta innovación se basó en el modelo instruccional de las 5E (Enganchar, Explorar, Explicar, Elaborar, Evaluar), que según explica Bybee (2002) permite resolver problemas al estudiante enfocándose en su propio aprendizaje. De acuerdo al autor, este modelo en referencia al diseño instruccional tiene como pasos: 1) enganchar la lección; 2) explorar la lección; 3) explicar la lección; 4) elaborar la lección y, 5) evaluar la lección.

Como instrumentos de evaluación de los conocimientos se emplearon pruebas estandarizadas de pretest y post test adaptadas del examen KET de la Universidad de Cambridge que en tres secciones miden el conocimiento de las habilidades lingüísticas de Reading y Writing, Listening y Speaking. La sección Reading y Writing del examen KET estandarizado está compuesta por 9 partes de las cuales se tomaron para la prueba adaptada las partes 1, 2, 3, y 4. La sección Listening está compuesta por 5 partes de las cuales se tomaron para la prueba adaptada las partes 1, 2 y 3. La sección Speaking está compuesta por 2 partes de las cuales se tomó solo la parte 1 para la prueba adaptada.

La prueba de pretest y post test tuvo una ponderación de 10 puntos. Se estableció una escala de valoración por rangos de acuerdo a puntos obtenidos: excelente $=10-9.6$, muy bueno $=$ 9.5-8.5, bueno $=8.4-7.5$, regular $=7.4-6.5$, deficiente $=6.4-0$. Se diseñó una rúbrica para la parametrización de esta escala con criterios de logros. Se evaluaron 3 aspectos del aprendizaje en las pruebas así como en las actividades de clase: estructura gramatical, correcto uso del lenguaje y vocabulario.

\section{Resultados}

El siguiente cuadro muestra los resultados del pretest tanto del grupo de control como del grupo experimental por número de estudiantes de acuerdo a la escala de valoración por 
rangos en cada uno de los tres aspectos evaluados: estructura gramatical, correcto uso del lenguaje y vocabulario.

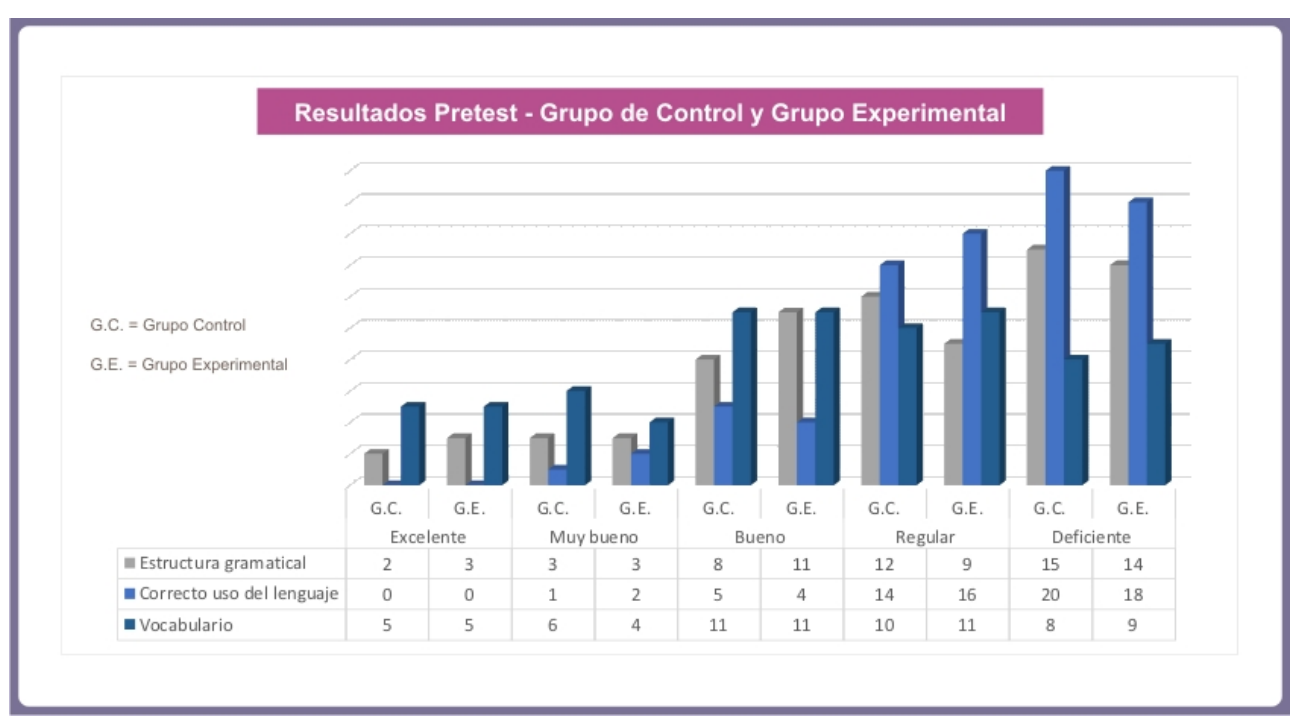

Fig. 4 Resultados del Pretest

Los resultados del pretest en lo que concierne a estructura gramatical arrojaron que en cuanto al grupo de control, $5 \%$ de los estudiantes se ubicaron en el rango de excelente, $27.5 \%$ en el rango de entre muy bueno y bueno; y $67.5 \%$ en el rango de entre regular y deficiente. En cuento al grupo experimental, $7.5 \%$ de los estudiantes se ubicaron en el rango de excelente, $35 \%$ en el rango de entre muy bueno y bueno; y 57.5\% en el rango de entre regular y deficiente.

Los resultados del pretest en lo que concierne a correcto uso del lenguaje para el grupo de control determinó que $15 \%$ de los estudiantes se ubicaron en el rango de entre muy bueno y bueno, y $85 \%$ en el rango de entre regular y deficiente. En cuento al grupo experimental, $15 \%$ de los estudiantes se ubicaron entre muy bueno y bueno; y $85 \%$ entre regular y deficiente. No hubo estudiantes en el rango de excelente para este aspecto.

Por último, los resultados del pretest para el aspecto de vocabulario determinó para el grupo de control que $12.5 \%$ de los estudiantes se ubicaron en el rango de excelente, $42.5 \%$ entre muy bueno y bueno; y $45 \%$ en el rango de entre regular y deficiente. Por su lado, el grupo experimental tuvo resultados en este aspecto de $12.5 \%$ de los estudiantes en el rango de excelente, $37.5 \%$ entre muy bueno y bueno; y $50 \%$ en el rango de entre regular y deficiente.

El pretest indicó que al iniciar el curso los alumnos de ambos grupos considerando los rangos regular y deficiente mostraron deficiencias en los conocimientos en lo que respecta a estructura gramatical en más de un $60 \%$, correcto uso del lenguaje en un $85 \%$; y vocabulario en cerca de un $48 \%$.

El siguiente cuadro muestra los resultados del post test tanto del grupo de control como del grupo experimental en los tres aspectos evaluados: estructura gramatical, correcto uso del lenguaje y vocabulario. 


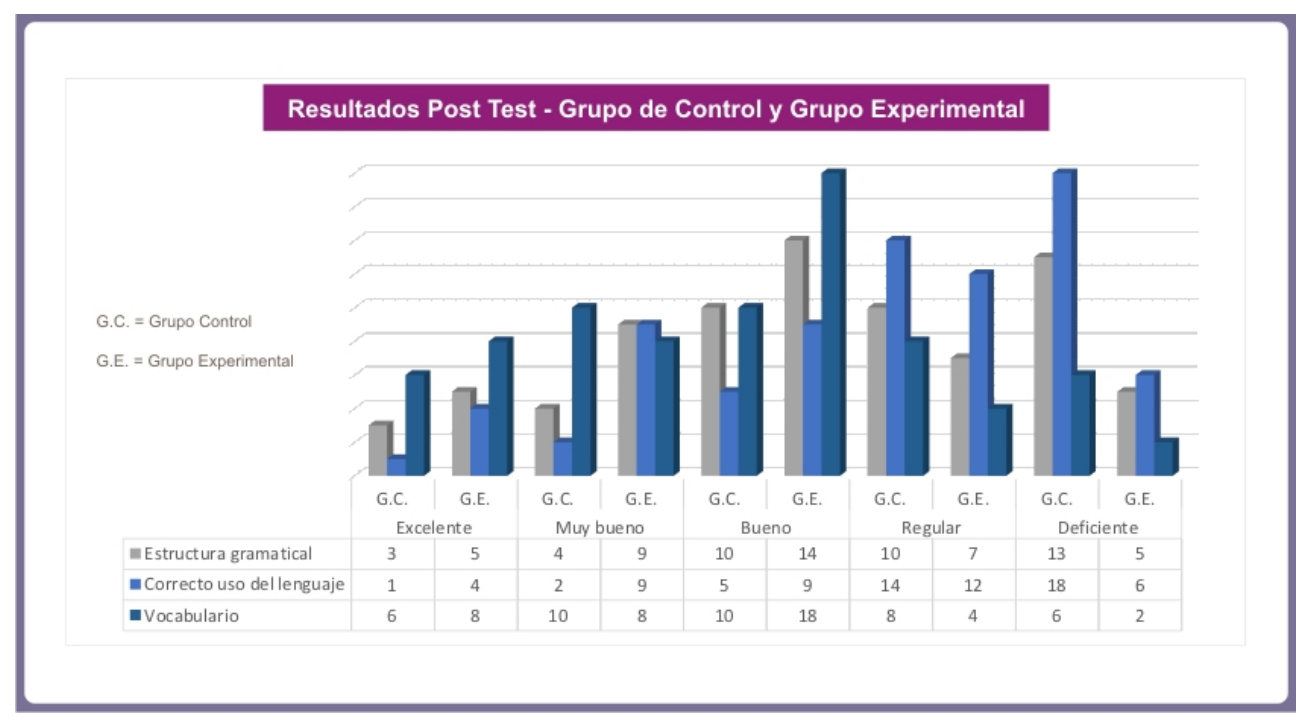

Fig. 5 Resultados del Post Test

Los resultados del post test evidenciaron aumentos y reducciones porcentuales con respecto a los resultados del pretest. El grupo de control en el aspecto de estructura gramatical registró cambios de +2.5 excelente, +2.5 muy bueno, +5 bueno, -5 regular, -5 deficiente. El grupo experimental logró cambios de +5 excelente, +15 muy bueno, +10 bueno, -5 regular, -25 deficiente.

El grupo de control en el aspecto de correcto uso del lenguaje registró cambios de +2.5 excelente, +2.5 muy bueno, 0 bueno, 0 regular, -5 deficiente. El grupo experimental logró cambios de +10 excelente, +17.5 muy bueno, +20 bueno, -10 regular, -37.5 deficiente.

El grupo de control en el aspecto de vocabulario registró cambios de +2.5 excelente, +10 muy bueno, -2.5 bueno, -5 regular, -5 deficiente. El grupo experimental logró cambios de +7.5 excelente, +10 muy bueno, +22.5 bueno, -17.5 regular, -22.5 deficiente.

El post test indicó diferencias considerables en el mejoramiento de todos los aspectos que lograron los participantes del grupo experimental en relación a los resultados del pretest. Persistieron deficiencias en el grupo de control. El siguiente cuadro muestra porcentualmente los resultados tanto del pretest como del post test. 


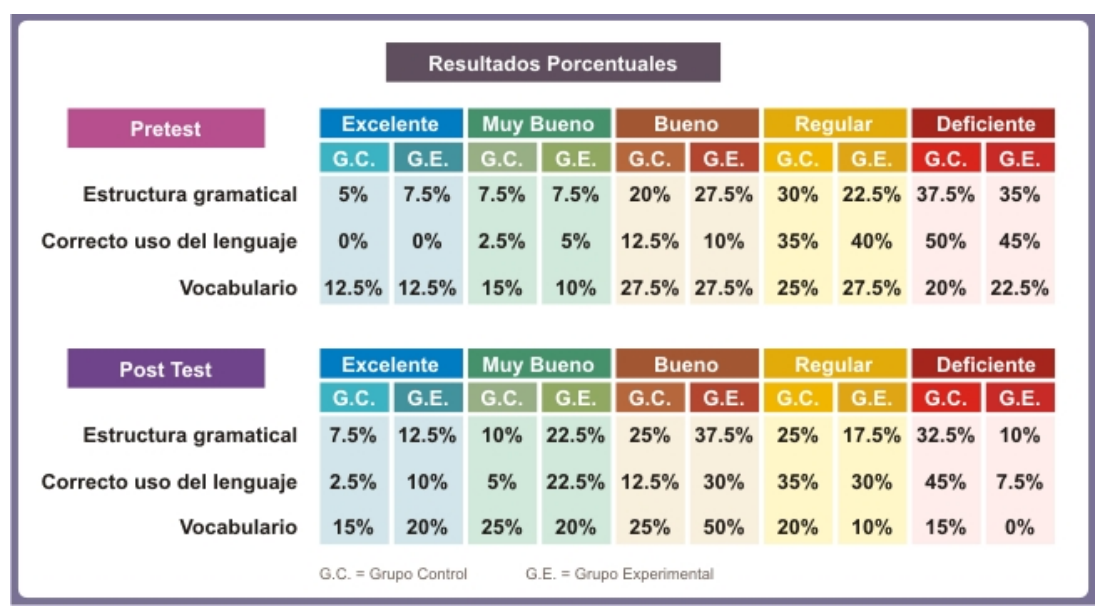

Fig. 6 Resultados porcentuales Pretest y Post Test

\section{Conclusiones}

Reducir el tamaño de la clase de la asignatura de Inglés I fue satisfactoriamente posible, en concordancia al objetivo establecido. Este estudio comprobó positivamente las tres hipótesis planteadas.

Se comprobó como muy beneficiosa la reducción del tamaño de la clase de Inglés empleando la modalidad Blended Learning, que para los participantes del grupo experimental significó una novedad en la que según ellos a través de una encuesta de opinión realizada, indicaron que estudiar en base a su tiempo y a su comodidad aportó a tener mejor desempeño. El hecho de contar con videos tutoriales, ejercicios orales y escritos a su completa disposición en un formato más dinámico tanto en forma virtual como presencial les permitió estar en ventaja con el grupo de control. Los resultados demuestran que fue superior el desempeño del grupo experimental en relación al grupo de control teniendo como indicadores destacados de dicho desempeño: mejora progresiva en la producción oral y la pronunciación, así como la comprensión auditiva comprobando que la modalidad Blended Learning puede reforzar de mejor forma el aprendizaje del idioma Inglés en comparación al aprendizaje presencial que para este estudio demostró como incidencia el inferior desempeño que lograron los estudiantes del grupo de control que según lo expresado por su profesor no promovió el trabajo autónomo ni el trabajo en equipo como se hubiera querido.

Otras de las validaciones importantes del estudio fue determinar que para poder reducir el tamaño de una clase empleando la modalidad Blended Learning es indispensable el uso de recursos tecnológicos educativos de apoyo como fue el caso de las aplicaciones NOMON PILLBOOK y NOMON CISE, ya que entre sus bondades de uso aplicado está el dar un marco de ordenamiento a la secuencia metodológica de la clase, así también como un recurso optimizador del esfuerzo del profesor y los estudiantes que permitió que dicho esfuerzo cognitivo hecho estuviese centrado en el aprendizaje, de allí la posibilidad de reducir el tiempo de la clase sin afectar la calidad del aprendizaje. 
Reducción del tamaño de la clase de inglés empleando Blended Learning como estrategia de aprendizaje

De parte de los profesores que participaron en el estudio se trató siempre en la medida posible de evitar el cometimiento de sesgos en la aplicación de ambos métodos. Al final del experimento, por parte de ambos profesores se concluyó que las clases reducidas pueden mejorar aspectos como la motivación, la objetividad y el trabajo colaborativo. Así mismo, esta discusión determinó también como aspectos negativos o amenazantes que deben considerarse para las clases reducidas empleando Blended Learning a aspectos como que el tiempo presencial destinado podría resultar muy limitado para ciertos estudiantes que requieren mayor apoyo tutorial; y que el profesor hace mayores gastos de esfuerzo en comparación a las clases tradicionales no reducidas.

Otro de los aspectos de consideración que surgieron de este estudio: es la posición de la institución educativa, ya que realizar una innovación de este tipo involucra asumir nuevos roles metodológicos que intervengan paradigmas vigentes del aprendizaje tradicional. Esto también se refiere a la inversión de recursos y esfuerzos que de no realizarse adecuadamente habrían afectado la calidad del experimento.

Se recomienda repetir el experimento con nuevas poblaciones estudiantiles ya sea con la misma asignatura o su asignatura post-requisito para la comparación y validación de resultados.

\section{Bibliografía}

Biddle, B., \& Berliner, D. (2008). Small Classes and its effects.

Burbules, N. (2012). El aprendizaje ubicuo y el futuro de la enseñanza.

Encounters/Encuentros/Rencontres on Education Vol. 13, 3-14.

Bybee, R. (2002). Learning Science and the Science of Learning. NSTA Press.

Calero, J., \& Escardibul, J. (2007). Evaluación de servicios educativos: El rendimiento en los centros públicos y privados medido en Pisa-2003. Barcelona: Treball 2007.

Chingos, M. (2013). Tamaño de la clase y resultados de los estudiantes: investigación e implicaciones politicas. Ponencia en el Instituto Nacional de Evaluación Educativo.

Council for Cultural Cooperation Education Committee. (2002). Common European Framework for

Languages: Learning, Teaching, Assessment. Strasbourg: Language Policy Division.

Cronquist, K., \& Fiszbein, A. (2017). El aprendizaje del inglés en América Latina. El Diálogo

Liderago para las Américas.

Garrido, C., \& Pernas, M. (2005). El aprendizaje del idioma inglés en las carreras de Ciencias Médicas. Instituto Superior de Ciencias Médicas de La Habana.

Hans, A., \& Hans, E. (2015). A Comparative Study of English for Specific Purposes (ESP) and English as a Second Language (ESL) Programs. International Journal on Studies in English Language and Literature (IJSELL), 26-31.

Hughes, L. (2005). Current issues in cchool leadership. Mahwah, New Jersey: Lawrence Erlbaum Associates, Publishers.

Instituto Cervantes. (2012). MArco Común Europeo de Referencia para las Lenguas: Aprendizaje, Enseñanza, Evaluación. Madrid: Artes Gráficas Fernandez.

(cc) EY-NC-ND 2018, Universitat Politècnica de València

Congreso IN-RED (2018) 
Mayer, S., \& Peterson, P. (1999). Earning and Learning, How schools matter. Washington D.C.: Brookings Institution Press.

Mora, J. (2015). Hacia un curriculum alternativo para la enseñanza del inglés a nivel universitario. Bogota: Universidad Militar Nueva Granada.

Mosteller, F. (1995). The Tennessee Study of Class Size in the Early School Grades. The Future of Children Critical Issues for Children and Youths Vol. 5 • No. 2, 113-127.

OECD. (2011). Education at a Glance 2011: OECD Indicators. OECD Publishing. Obtenido de http://dx.doi.org/10.1787/eag-2011-en

Singh, H. (2003). Building Effective Blended Learning Programs . Issue of Educational Technology, Volume 43, Number 6, 51-54.

SISTEMA DE ENSEÑANZA NOMON ENGLISH LEARNING, Carrasco C. (2014) IEPI.

Vera, F. (2008). La modalidad Blended Learning en Educación Superior. Rancagua, Chile. 